\title{
Manejo de resíduos da insulinoterapia produzidos no domicílio por indivíduos com diabetes mellitus no contexto da atenção básica à saúde
}

\author{
Management of insulin therapy residues produced at home by individuals with diabetes \\ mellitus in the context of primary health care
}

Manejo de los residuos de la terapia con insulina producidos en el hogar por personas con diabetes mellitus en el contexto de la atención primaria de salud

Taysa Cristina Cardoso Freitas ${ }^{1}$, Mariana Stefany Cardoso Nascimento ${ }^{2}$, Sibelle Gonçalves de Almeida $^{2}$, Brunna Thaís Costa ${ }^{2}$, Thamires Dias de Carvalho², Andreia Correia ${ }^{2}$, Joice Fernanda Costa Quadros $^{3}$, Helio Alves da Silva ${ }^{1 *}$, Gizele Freitas Rodrigues ${ }^{2}$, Richard Rennan Soares Barbosa².

\section{RESUMO}

Objetivo: Conhecer como ocorre o manejo de resíduos da insulinoterapia produzidos no domicílio de indivíduos com diabetes mellitus no contexto da atenção básica à saúde. Métodos: Trata-se de um estudo de revisão integrativa de literatura a partir das bases de dados Literatura Latino-Americana e do Caribe em Ciências da Saúde (LILACS), Scientific Electronic Library Online (Scielo) e da Biblioteca Virtual em Saúde (BVS), foram utilizados os descritores "diabetes mellitus", "insulina" e "gerenciamento de resíduos" para guiar a busca. Resultados: Não foram encontradas iniciativas e programas instituídos de educação em saúde para o gerenciamento adequado dos resíduos gerados pelos usuários insulinodependentes, sendo também a temática pouco explorada na literatura. Considerações finais: Os resultados obtidos na presente investigação evidenciam que não há manejo correto de resíduos da insulinoterapia produzidos pelos indivíduos com diabetes mellitus no contexto dos serviços de atenção básica à saúde de forma geral, sendo as ações realizadas de forma pontual e assistemática.

Palavras-chave: Diabetes mellitus, Insulina, Gerenciamento de resíduos.

\begin{abstract}
Objective: To understand how insulin therapy waste is produced in the home of individuals with diabetes mellitus in the context of primary health care. Methods: This is an integrative literature review study based on the Latin American and Caribbean Literature in Health Sciences (LILACS), Scientific Electronic Library Online (SciELO) and the Virtual Health Library (VHL) databases, the descriptors "diabetes mellitus", "insulin" and "waste management" were used to guide the search. Results: No instituted health education initiatives and programs were found for the proper management of waste generated by insulin-dependent users, and the theme is also little explored in the literature. Final considerations: The results obtained in the present investigation show that there is no correct management of insulin therapy residues produced by individuals with diabetes mellitus in the context of primary health care services in general, with the actions being carried out in a punctual and unsystematic manner.
\end{abstract}

Key words: Diabetes mellitus, Insulin, Waste management.

\footnotetext{
${ }^{1}$ Faculdade Santo Agostinho (FASA), Montes Claros - MG. *E-mail: scmenfermagem9@gmail.com.

2 Faculdades Unidas do Norte de Minas Gerais (FUNORTE), Montes Claros - MG.

${ }^{3}$ Faculdade de Saúde Ibituruna (FASE), Montes Claros - MG.
} 


\section{RESUMEN}

Objetivo: Comprender cómo se producen los residuos de la terapia con insulina en el hogar de personas con diabetes mellitus en el contexto de la atención primaria de salud. Métodos: Se trata de un estudio de revisión integradora de la literatura basado en las bases de datos de Literatura Latinoamericana y Caribeña en Ciencias de la Salud (LILACS), Biblioteca Electrónica Científica en Línea (SciELO) y Biblioteca Virtual en Salud (BVS), los descriptores "diabetes mellitus", "insulina "Y" gestión de residuos "se utilizaron para guiar la búsqueda. Resultados: No existieron iniciativas y programas de educación en salud instituidos para el manejo adecuado de los desechos generados por los usuarios insulinodependientes, y el tema también está poco explorado en la literatura. Consideraciones finales: Los resultados obtenidos en la presente investigación muestran que no existe un manejo correcto de los residuos de la terapia con insulina producidos por personas con diabetes mellitus en el contexto de los servicios de atención primaria de salud en general, realizando se las acciones de manera puntual y no sistemática.

Palabras clave: Diabetes mellitus, Insulina, Gestión de residuos.

\section{INTRODUÇÃO}

O Diabetes Mellitus (DM) é um conjunto de doenças metabólicas em que ocorre o aumento nos níveis de glicemia sanguínea e desordem no metabolismo de carboidratos, proteínas e gorduras, associando-se a disfuncionalidades e insuficiência de órgãos, sobretudo olhos, rins, nervos, cérebro, coração e vasos sanguíneos. É resultado de falhas na secreção ou efeito inadequado da insulina, causando a destruição das células beta do pâncreas e/ou o processo de resistência insulínica, dentre outros fatores (BRASIL, 2013).

No ano de 2015 , cerca de $8,8 \%$ da população do planeta com idade entre 20 a 79 anos apresentava o DM. A projeção é que no ano de 2040 este quantitativo eleve-se para mais de 642 milhões de indivíduos acometidos com essa doença no mundo (IDF, 2015). O DM apresenta altas taxas de incidência e conjuntamente altas taxas de mortalidade precoce. Cerca de 5 milhões de indivíduos com idade entre 20 e 79 anos morreram no ano de 2015, o que responde por 6 mortes a cada seis segundos (SBD, 2017).

O DM vem crescendo sua relevância pelo aumento da prevalência e relação com dislipidemias, hipertensão arterial sistêmica e distúrbio endotelial. A DM apresenta-se sem sintomas na grande maioria dos casos e em algumas ocasiões, o diagnóstico é realizado inicialmente por meio da detecção de complicações crônicas. As opções terapêuticas disponíveis para o tratamento da doença podem ser medidas farmacológicas e não farmacológicas (SBD, 2015).

Nesse contexto, a insulinoterapia é um dos recursos terapêuticos de maior relevância para os indivíduos com DM e também o que demanda mais cuidados. Além de cautela quanto ao processo para administração, a insulinoterapia é fonte de resíduos, os quais podem ser desde agulhas, algodão para ser utilizado após o procedimento, fitas reagentes e por fim seringas e lancetas (ADA 2013; BOAS LCG, et al. 2014).

Considerando que os usuários com diabetes podem realizar inúmeras aplicações de insulina rotineiramente e verificar a glicemia capilar também de forma habitual, estes usuários devem ser orientados em relação à administração e descarte de resíduos. Devido a serem procedimentos invasivos, independentemente do local da assistência, existe risco relacionado ao cuidado em saúde (ALVES SB, 2012). Resíduos resultantes dos serviços de assistência à saúde humana e/ou animal, o que inclui, o domicílio, são denominados como Resíduos de Serviços de Saúde (RSSs) (BRASIL, 2012).

Essa modalidade de resíduo necessita de tratamento diferenciado em todas as fases de manejo, pois se trata de componentes químicos, biológicos e físicos que causam danos ao meio ambiente e aos indivíduos (ANDRÉ SCS e TAKAYANAGUI AMM, 2015). Os RSSs são divididos em cinco grupos diferentes, ou seja, grupo A (potencialmente infectantes); Grupo B (químicos); grupo C (radioativos); grupo D (comuns) e grupo $\mathrm{E}$ (perfurocortantes). O gerenciamento dos resíduos deve obedecer às fases de segregação, acondicionamento, identificação, transporte interno, armazenamento em caráter temporário, tratamento, armazenagem externa e disposição final (BRASIL, 2012). Os resíduos gerados pela insulinoterapia 
produzidos na assistência no domicílio, constantes nos grupos A, B e, devem ser armazenados e coletados por pessoas habilitadas para a tarefa e conduzidos a instituições de assistência à saúde para que estas façam o encaminhamento adequado (ALVES SB, 2012).

Para os serviços de saúde o descarte de resíduos escarificantes é organizado pela Agência Nacional de Vigilância Sanitária (ANVISA) - entidade que responde pela regulamentação do descarte dos RSS - a qual determina por meio da Resolução da Diretoria Colegiada (RDC) № 306/04 que os serviços disponham do Plano de Gerenciamento de Resíduos de Serviço de Saúde (PGRSS (VAZ KV, et al., 2011). Entretanto, para a população que produz esse material no domicílio não existe legislação específica. Em razão à omissão legal e a falta de programas de gerenciamento, muitos pacientes acabam por descartar os resíduos produzidos em locais inadequados (SANTOS LN e RUIZ JB, 2020).

O primeiro passo do manejo dos resíduos é a segregação, apontada como a etapa mais importante. Portanto a segregação inadequada provoca prejuízos resultantes do aumento no custo para o descarte dos resíduos, possibilitando assim, risco para os trabalhadores e usuários, como também danos ambientais (ALVES SB, 2012).

A ausência desse gerenciamento dos RSSs, conjuntamente com a frequência em que os trabalhadores são expostos ao material biológico, expõe o alto risco para doenças transmitidas por sangue ou por fluidos corpóreos e acidentes, além dos próprios usuários (RAMOS YS, et al., 2011).

Nessa discussão, faz-se imprescindível o desenvolvimento educacional, administrativo e até mesmo de meios legislativos para o adequado gerenciamento dos RSSs que são resultados do cuidado a nível domiciliar. É claro a exigência de um plano de estratégico para prevenção de acidentes e preservação ambiental (VIEIRA M e PADILHA MICS, 2008).

Assim, o presente estudo busca conhecer como ocorre o manejo de resíduos da insulinoterapia produzidos no domicílio de indivíduos com diabetes mellitus no contexto da atenção básica à saúde.

\section{MÉTODOS}

Trata-se de um estudo de revisão integrativa da literatura. Para a realização da revisão integrativa foram seguidas seis fases interdependentes: 1) elaboração da pergunta norteadora; 2) busca na literatura; 3) coleta de dados; 4) análise crítica dos estudos incluídos; 5) discussão dos resultados e 6) apresentação da revisão integrativa (URSI ES, 2005).

Considerou-se como questão norteadora: Como ocorre o manejo de resíduos da insulinoterapia produzidos no domicílio de indivíduos com diabetes mellitus no contexto da atenção básica à saúde?

O levantamento das publicações foi realizado entre os meses de junho a janeiro de 2021, a partir das bases de dados LILACS, Scielo e Biblioteca Virtual em Saúde (BVS). Para a coleta de dados foi utilizado instrumento validado por Ursi ES (2005) com as seguintes informações: dados de identificação do artigo (título, autores, nome do periódico, ano de publicação, volume e número), tipo de estudo, local de estudo, objetivo do estudo, eixo temático, classificação Qualis/Capes, periódico de publicação, nível de evidência, desfechos, dentre outros. A seleção dos artigos foi realizada de forma independente pelos pesquisadores, as divergências foram resolvidas com o uso do instrumento validado.

Foram utilizados como descritores "Diabetes Mellitus", "Insulina " e "Gerenciamento de Resíduos", utilizando o operador booleano "and" para combinação. Dessa forma, foram identificadas inicialmente 20 publicações elegíveis para a inclusão nesta revisão.

Para selecionar as publicações foram estabelecidos critérios de inclusão: textos completos, disponibilidade do artigo completo e que abordassem a temática, textos redigidos em português, inglês ou espanhol em um corte temporal de 12 anos. Foi adotada essa temporalidade em razão da baixa produção sobre o objeto de estudo. Todos os artigos selecionados pela estratégia de busca proposta foram analisados pela leitura completa. Foram excluídos os estudos que não atendiam as exigências anteriores e aqueles que não 
abordaram o tema de pesquisa, resultando em 10 publicações selecionadas, por atenderem os critérios anteriormente descritos (Figura 1).

Figura 1 - Fluxo de amostragem dos artigos.

Fase 1: Definição das bases de dados

Fase 2: Definição dos descritores

Fase 3: Análise dos títulos e resumos

Fase 4: Análise dos textos completos

Revisão integrativa de literatura

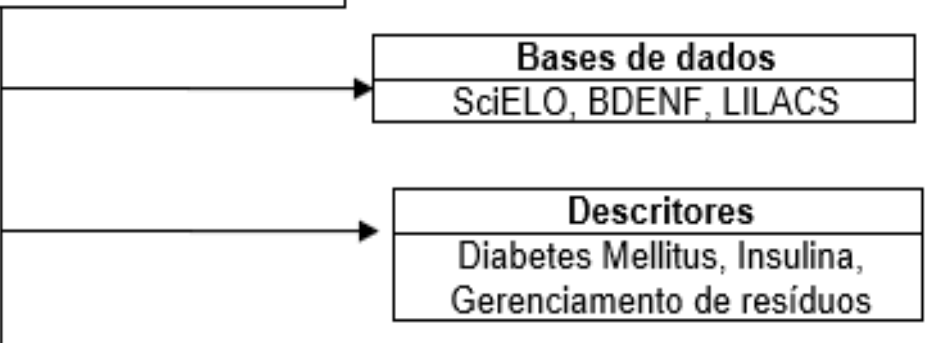

54 artigos potencialmente relevantes.

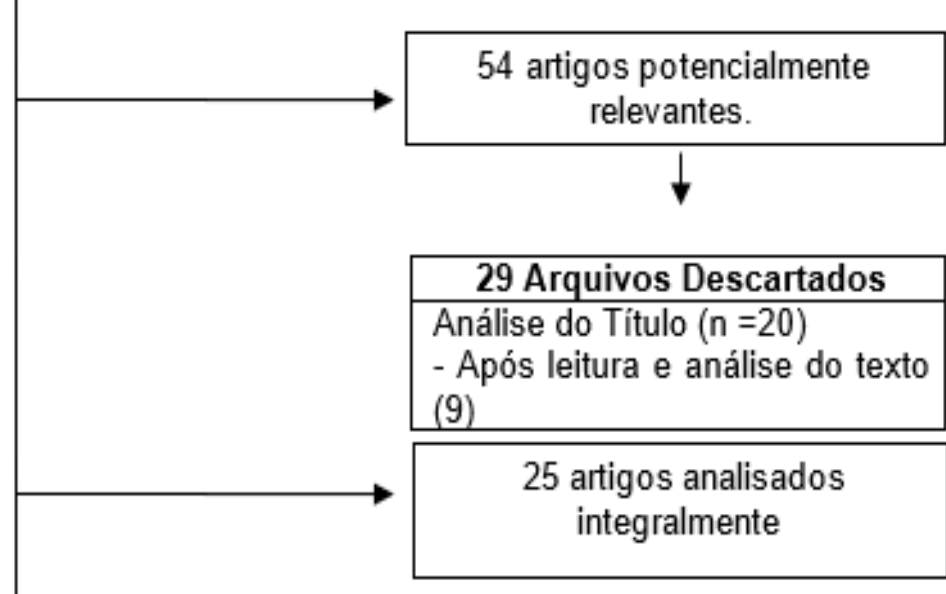

10 artigos incluídos no Estudo

Fonte: Freitas TCC, et al., 2021.

\section{RESULTADOS}

Apresenta-se a síntese das principais características dos artigos incluídos no estudo dispostos em ordem decrescente de acordo com o ano, do mais recente ao mais antigo. Para a melhor compreensão os artigos foram organizados de acordo com o ano de publicação, título, periódico de publicação, delineamento, objetivo e principais achados (Quadro 1). 
Quadro 1 - Artigos selecionados para análise integral.

\begin{tabular}{|c|c|c|c|c|c|}
\hline Autor(es)/Ano & Título & Periódico & Delineamento & Objetivo & Desfecho \\
\hline $\begin{array}{l}\text { Santos TNL, et } \\
\text { al. (2019) }\end{array}$ & $\begin{array}{l}\text { Resíduos de saúde nos } \\
\text { domicílios de pacientes } \\
\text { com diabetes mellitus. }\end{array}$ & $\begin{array}{l}\text { Revista } \\
\text { Laborativa. }\end{array}$ & $\begin{array}{l}\text { Estudo } \\
\text { quantitativo, } \\
\text { transversal, } \\
\text { descritivo. }\end{array}$ & $\begin{array}{l}\text { Identificar o gerenciamento dos } \\
\text { resíduos de saúde nos domicílios } \\
\text { de pacientes com diabetes } \\
\text { mellitus acompanhados nos } \\
\text { centros de saúde de referência } \\
\text { do município de Campos dos } \\
\text { Goytacazes/RJ. }\end{array}$ & $\begin{array}{l}\text { Os resíduos são gerados com frequência nos } \\
\text { domicílios sendo a maioria descartada de } \\
\text { forma inadequada. } \\
\text { Houve um predomínio do relato de ausência } \\
\text { de orientação a respeito do gerenciamento } \\
\text { adequado. }\end{array}$ \\
\hline $\begin{array}{l}\text { Aquino S, et al. } \\
(2019)\end{array}$ & $\begin{array}{l}\text { Percepção de diabéticos e } \\
\text { papel dos profissionais de } \\
\text { saúde sobre a educação } \\
\text { ambiental de resíduos } \\
\text { perfurocortantes } \\
\text { produzidos em domicílios. }\end{array}$ & $\begin{array}{l}\text { Revista brasileira } \\
\text { de educação } \\
\text { ambiental. }\end{array}$ & $\begin{array}{ll}\text { Estudo } & \text { de } \\
\text { abordagem } & \\
\text { mista, } & \text { de } \\
\text { natureza } & \\
\text { descritiva } & \text { e } \\
\text { exploratória } & \end{array}$ & $\begin{array}{l}\text { Analisar a percepção de } \\
\text { diabéticos insulinodependentes } \\
\text { sobre a responsabilidade } \\
\text { ambiental da geração de seu } \\
\text { resíduo. }\end{array}$ & $\begin{array}{l}\text { Existe uma inadequação na educação sobre } \\
\text { o manejo e descarte dos resíduos oriundos } \\
\text { do tratamento e monitoramento de diabéticos } \\
\text { em domicílio, sendo o destino de materiais } \\
\text { como fitas reagentes (com sangue), } \\
\text { lancetas, seringas e agulhas usadas } \\
\text { descartados em lixo doméstico comum ou } \\
\text { em garrafas plásticas (PET), encaminhando, } \\
\text { posteriormente, para algum serviço de } \\
\text { saúde. }\end{array}$ \\
\hline $\begin{array}{l}\text { Santos LN, Ruiz } \\
\text { JB. (2020) }\end{array}$ & $\begin{array}{l}\text { Caracterização } \text { e } \\
\text { quantificação dos resíduos } \\
\text { perfurocortantes gerados } \\
\text { por diabéticos do } \\
\text { município de Umuaram, } \\
\text { PR, Brasil. }\end{array}$ & $\begin{array}{l}\text { Ciência \& Saúde } \\
\text { Coletiva. }\end{array}$ & $\begin{array}{l}\text { Pesquisa de } \\
\text { abordagem } \\
\text { quantitativa. }\end{array}$ & $\begin{array}{l}\text { Caracterizar e quantificar os } \\
\text { resíduos perfurocortantes } \\
\text { gerados por diabéticos } \\
\text { insulinodependentes } \\
\text { frequentam unidades de saúde } \\
\text { da família do município de } \\
\text { Umuarama. }\end{array}$ & $\begin{array}{l}\text { Os resíduos eram armazenados com maior } \\
\text { prevalência - em sacolas plásticas } \\
(20,80 \%) \text { garrafas PET, frascos de } \\
\text { amaciante/maionese, etc. ( } 46,98 \%) \text {. O lixo } \\
\text { doméstico comum foi o principal local de } \\
\text { descarte dos perfurocortantes. Aqueles } \\
\text { usuários que não receberam informações } \\
\text { sobre o manejo dos resíduos os desprezam } \\
\text { de maneira incorreta. }\end{array}$ \\
\hline $\begin{array}{l}\text { Silva ENSF, et al. } \\
(2013)\end{array}$ & $\begin{array}{l}\text { Descarte de seringas e } \\
\text { agulhas por pacientes com } \\
\text { diabetes mellitus. }\end{array}$ & $\begin{array}{l}\text { Revista } \\
\text { Enfermagem } \\
\text { Contemporânea. }\end{array}$ & $\begin{array}{l}\text { Pesquisa } \\
\text { qualitativa } \\
\text { descritiva. }\end{array}$ & 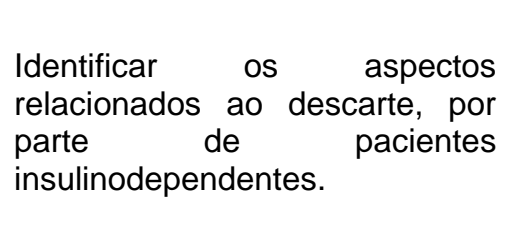 & $\begin{array}{l}\text { Verificou-se um déficit de informações sobre } \\
\text { os aspectos envolvidos no destino final do } \\
\text { perfurocortante. O processo educativo } \\
\text { possibilita um maior nível de conhecimento } \\
\text { promove esclarecimentos sobre o descarte } \\
\text { de forma adequada dos perfurocortantes. }\end{array}$ \\
\hline
\end{tabular}




\begin{tabular}{|c|c|c|c|c|c|}
\hline Autor(es)/Ano & Título & Periódico & Delineamento & Objetivo & Desfecho \\
\hline $\begin{array}{l}\text { Santos RDT, et } \\
\text { al. }(2020)\end{array}$ & $\begin{array}{ll}\text { Descarte residencial } & \text { de } \\
\text { resíduos } & \text { de } \\
\text { insulinoterapia } & \text { no } \\
\text { município de Juazeirinho, } \\
\text { Paraíba. }\end{array}$ & $\begin{array}{l}\text { Revista } \\
\text { Interdisciplinar } \\
\text { em Saúde, }\end{array}$ & $\begin{array}{l}\text { Estudo de } \\
\text { prevalência, } \\
\text { com } \\
\text { abordagem } \\
\text { quantitativa. }\end{array}$ & $\begin{array}{l}\text { Estudo de prevalência, com } \\
\text { abordagem quantitativa. }\end{array}$ & $\begin{array}{l}\text { Foi mais relatado o uso de garrafas PET, } \\
\text { entretanto, muitos relataram apenas o uso de } \\
\text { sacos plásticos comuns, realizando o } \\
\text { descarte final junto aos demais resíduos } \\
\text { residenciais para a coleta de lixo municipal. }\end{array}$ \\
\hline $\begin{array}{l}\text { Alves SB, et al. } \\
(2012)\end{array}$ & $\begin{array}{l}\text { Manejo de resíduos } \\
\text { gerados na assistência } \\
\text { domiciliar pela Estratégia } \\
\text { de Saúde da Família. }\end{array}$ & $\begin{array}{l}\text { Revista Brasileira } \\
\text { de Enfermagem. }\end{array}$ & $\begin{array}{l}\text { Tratou-se de } \\
\text { uma pesquisa } \\
\text { descritiva, com } \\
\text { abordagem } \\
\text { Quantitativa. }\end{array}$ & $\begin{array}{l}\text { Analisar o manejo dos resíduos } \\
\text { gerados pela assistência } \\
\text { domiciliar. }\end{array}$ & $\begin{array}{l}\text { Foram observadas inadequações que estão } \\
\text { relacionadas às dificuldades inerentes ao } \\
\text { manejo dos resíduos de serviços de saúde, } \\
\text { mas também às características diferenciadas } \\
\text { da assistência no domicílio. }\end{array}$ \\
\hline $\begin{array}{l}\text { André SCS, } \\
\text { Takayanagui } \\
\text { AMM (2015) }\end{array}$ & $\begin{array}{l}\text { Orientações sobre o } \\
\text { descarte de resíduos } \\
\text { gerados em domicílios de } \\
\text { usuários de insulina. }\end{array}$ & $\begin{array}{ll}\text { Revista } & \text { Baiana } \\
\text { de } & \text { Saúde } \\
\text { Pública. } & \end{array}$ & $\begin{array}{l}\text { Trata-se de um } \\
\text { estudo } \\
\text { descritivo e } \\
\text { exploratório. }\end{array}$ & $\begin{array}{l}\text { Identificar os tipos de orientação } \\
\text { recebidas por usuários de } \\
\text { insulina quanto ao descarte de } \\
\text { resíduos gerados pelo uso desse } \\
\text { medicamento e monitoramento } \\
\text { glicêmico em seus domicílios. }\end{array}$ & $\begin{array}{l}\text { Há necessidade de implantação de um } \\
\text { protocolo sobre o manejo de resíduos do } \\
\text { serviço de saúde em domicílio para } \\
\text { direcionar as ações e orientações aos } \\
\text { usuários de insulina. }\end{array}$ \\
\hline $\begin{array}{l}\text { Cunha GHD, et } \\
\text { al. }(2017)\end{array}$ & $\begin{array}{lr}\text { Resíduos } & \text { de } \\
\text { insulinoterapia produzidos } \\
\text { no domicílio de diabéticos } \\
\text { acompanhados na } \\
\text { Atenção Primária. }\end{array}$ & $\begin{array}{l}\text { Revista Brasileira } \\
\text { de Enfermagem. }\end{array}$ & $\begin{array}{l}\text { Estudo } \\
\text { transversal, } \\
\text { descritivo } \\
\text { quantitativo. }\end{array}$ & $\begin{array}{l}\text { Analisar o descarte de resíduos } \\
\text { da insulinoterapia produzidos no } \\
\text { domicílio de pessoas com } \\
\text { diabetes mellitus. }\end{array}$ & $\begin{array}{l}\text { Pacientes orientados sobre descarte tiveram } \\
21 \text { vezes mais chances de descartarem } \\
\text { resíduos adequadamente que os não } \\
\text { orientados. }\end{array}$ \\
\hline $\begin{array}{l}\text { Estequi JG, et al. } \\
\text { (2018) }\end{array}$ & $\begin{array}{l}\text { Resíduos gerados por } \\
\text { usuários de insulina em } \\
\text { domicílio. }\end{array}$ & $\begin{array}{l}\text { Revista Mineira } \\
\text { de Enfermagem. }\end{array}$ & $\begin{array}{l}\text { Estudo } \\
\text { descritivo e } \\
\text { exploratório, de } \\
\text { natureza } \\
\text { quantitativa }\end{array}$ & $\begin{array}{l}\text { Conhecer o manejo dos resíduos } \\
\text { gerados pelos usuários de } \\
\text { insulina em domicílio. }\end{array}$ & $\begin{array}{l}\text { O descarte incorreto de ao menos um dos } \\
\text { insumos utilizados, seja acondicionado em } \\
\text { recipiente rígido ou não, ocorreu tanto entre } \\
\text { os participantes que referiram ter recebido } \\
\text { informações sobre o descarte correto dos } \\
\text { RSS quanto entre os que não receberam. }\end{array}$ \\
\hline
\end{tabular}

Fonte: Freitas TCC, et al., 2021. 


\section{DISCUSSÃO}

A concepção de instrumentos e materiais revolucionários no campo da saúde é uma das grandes mudanças sucedidas na área da saúde no decorrer dos anos. Nesse campo, ressaltam-se os instrumentos e materiais descartáveis que promovem uma maior segurança do paciente, contudo, conjuntamente, acarretaram o crescimento da geração de resíduos (ESTIQUE JG, et al., 2018).

Em relação aos resíduos das instituições de saúde, frequentemente, preocupa-se de forma mais intensa com os produzidos por hospitais e clínicas em detrimento aos resíduos produzidos no contexto da atenção básica. A maior abrangência da Atenção Básica e as tarefas inerentes ao processo de trabalho das equipes, como por exemplo, a atenção domiciliar, tem acarretado novas necessidades a serem gerenciadas pelos profissionais de saúde. Nessa discussão, assume um papel de destaque, os usuários com doenças crônicas não transmissíveis (DCNT), sobretudo, o DM, que exige ainda o manejo dos RSSs resultados do processo de cuidado e autocuidado em nível domiciliar (TORRES HC, et al., 2014).

Nesse contexto, percebe-se a demanda de ampla produção científica voltada para questões e atitudes que orientam o saber e o fazer relacionado aos resíduos da assistência domiciliar, assim como trabalhos que possibilitam a abordagem de protocolos e práticas de manuseio de resíduos nesse setor, debatendo, sobretudo, o conteúdo na assistência na Estratégia Saúde da Família (ALVES SB, 2012). Ressalta-se que o manejo de resíduos resultantes da insulinoterapia é pouco explorado na literatura (CUNHA GH, et al., 2017).

No cotidiano das Unidades Básicas de Saúde, os usuários, frequentemente fazem uso de garrafas plásticas de água ou refrigerantes vazias, as quais são denominadas de garrafas de politereftalato de etileno (PET), contudo, esses recipientes não são os recomendados para o processo de descarte dos resíduos gerados pelos usuários que fazem uso de insulina, visto a fragilidade do material e o potencial para acidentes (BRASIL, 2013).

Em estudo realizado no Paquistão com 375 pacientes insulinodependentes, a maioria dos pacientes descartou os dispositivos usados (seringas, 92\%; canetas, 75\%; e lancetas, 91\%) em lixeira doméstica. Ademais, os pacientes que foram educados por um profissional médico ou sabiam ler/escrever foram menos propensos a descartar seringas e agulhas de forma inadequada (ISHTIAQ O, et al., 2012). Em outro ponto, deve-se salientar que o lixo gerado pelos usuários insulinodependentes e descartado de forma incorreta pode gerar riscos de acidentes aos profissionais de limpeza urbana (OURO K, 2011).

No Brasil, em inquérito conduzido no Paraná, os usuários relataram que após o uso, os resíduos eram armazenados com maior frequência, em sacolas plásticas $(20,80 \%)$, garrafas PET e frascos de amaciantes/maionese (46,98\%) (SANTOS LN, RUIZ JB, 2020).

Em outro estudo realizado um município de porte médio situado na região norte do Estado de Minas Gerais constatou-se que de 81 Unidades Básicas de Saúde que compunham a rede de atenção básica do município, apenas uma possuía o Plano de Gerenciamento de Resíduos de Serviços de Saúde (PAIVA PA, et al., 2017).

Dentre os resíduos produzidos por usuários com diagnóstico de DM em nível domiciliar, constatou-se a presença de canetas e frascos de insulina, agulhas, seringas, lancetas, algodão e fitas tipo reagentes. Calcula-se que há mais de 7,5 milhões de seringas utilizadas em nível residencial anualmente, sendo os usuários com diabetes os principais responsáveis por esse quantitativo, e este quantitativo não inclui o número de lancetas utilizadas por 25 milhões de indivíduos com diagnóstico médico de DM (GOLD K, 2011; ISHTIAQ O, et al., 2012).

A Resolução da Diretoria Colegiada (RDC) № 306 da Agência Nacional de Vigilância Sanitária (ANVISA) informa que é obrigatório a elaboração de um Plano de Gerenciamento de Resíduos de Serviços de Saúde (PGRSS) por todos os serviços de saúde que gerem resíduos. No PGRSS deve haver previsão para o manejo, segregação, acondicionamento, identificação, transporte interno, armazenamento temporário, tratamento, armazenamento externo, coleta, transporte para vias externas e por fim a destinação final (BRASIL, 2004). O Plano de gerenciamento é um documento de caráter institucional que deve basear-se nas singularidades de cada serviço produtor de resíduos, nesse plano, deve ser considerado os recursos materiais, possibilidades 
físicas, educação permanente dos profissionais, dentre outras variáveis que possam influenciar em alguma fase do gerenciamento (BRASIL, 2004).

De forma geral, de acordo com os estudos investigados os usuários insulinodependentes não faziam o manejo correto dos resíduos da insulinoterapia produzidos no domicílio, ressalta-se também que não foram encontrados iniciativas e programas instituídos de educação em saúde para o gerenciamento adequado dos resíduos gerados pelos usuários insulinodepentes, sendo também a temática pouco explorada na literatura. Este quadro é preocupante, visto o impacto da temática para os usuários, serviços de saúde, sociedade e meio ambiente.

O descarte das seringas e agulha acoplada deve ser feito em embalagem própria para material perfurocortante, devendo o mesmo ser ofertado pela unidade de saúde, podendo ser utilizado ainda uma embalagem que seja rígida e resistente, como por exemplo, um recipiente vazio de amaciantes de roupas. Quando esse recipiente estiver cheio, o mesmo deve ser encaminhado à unidade de saúde, para que dessa maneira, na Unidade Básica de Saúde seja realizado o descarte correto (BRASIL, 2013).

A educação apropriada sobre o descarte correto de perfurocortantes deve ser parte integrante do aconselhamento para os pacientes diabéticos. Médicos, enfermeiros e farmacêuticos devem assumir a responsabilidade de educar e reforçar as informações sobre os métodos corretos de descarte de materiais cortantes. Os pacientes devem ser aconselhados a descartar os objetos perfurocortantes em recipientes resistentes a perfurações e devolvê-los em recipientes seguros para descarte nas instituições distribuidoras. Os pacientes também devem ser informados sobre os riscos à saúde associados às agulhas usadas (GOVENDER D, et al., 2012). Frente às premissas, ressalta-se que os profissionais de enfermagem assumem papel de destaque no processo educativo dos usuários diabéticos (CUNHA GH, et al., 2017).

Nesse sentido, salienta-se que as orientações acerca dos cuidados com resíduos gerados no tratamento e controle do DM em nível domiciliar devem ser compreendidas na formação dos profissionais de saúde, sobretudo, dos enfermeiros que atuam no contexto da atenção básica, os quais assumem especial relevância, pois são os profissionais que frequentemente instruem os usuários de insulinoterapia no domicílio (CUNHA $\mathrm{GH}$, et al., 2017). Tendo em vista, esse propósito, é indispensável que os profissionais de saúde sejam rotineiramente atualizados acerca das melhores práticas de descarte dos RSSs, almejando a possibilidade de implementação das evidências cientificas a prática assistencial (ANDRÉ SCS e TAKAYANAGUI AMM, 2015).

Ainda acerca da orientação dos usuários com DM sobre os manejo e descarte dos RSSs produzidos em nível residencial, inquérito conduzido em Ribeirão Preto no Estado de São Paulo constatou que 61,5\% dos pacientes que faziam uso insulina em domicílio relataram terem recebido alguma modalidade de orientação sobre o descarte dos resíduos produzidos pela aplicação da insulina e/ou o processo de monitorização da glicemia. Destes, grande parte $(37,6 \%)$ relatou ter sido orientada por meio de profissionais que atuavam na farmácia da Unidade Básica de Saúde e 12,6\% por profissionais enfermeiros. Outra avaliação evidenciou que mais da metade dos usuários (51\%) tinham recebido orientações acerca do descarte dos RSSs nas consultas nas instituições de saúde, sendo que a maior parte (90\%) foi educado por enfermeiros, sendo o restante orientado por médicos (10\%) (CUNHA GH, et al., 2017).

Quando o usuário recebe orientações adequadas sobre o manejo dos resíduos ele apresenta maiores chances de realizar o descarte correto, diminuindo os riscos ambientais e acidentes na comunidade (SANTOS LN e RUIZ JB, 2020). A ausência de informação também foi encontrada em outras investigações (STACCIARINI TSG, et al., 2010; SILVA ÉNSF, et al., 2013; TAPIA CEV, 2009). No estado do Ceará, os achados de uma investigação evidenciaram que os usuários que receberam instrução formal acerca do manejo correto dos RSSs são 21 vezes mais inclinados a realizar o descarte de forma adequada (CUNHA $\mathrm{GH}$, et al., 2017).

Com o recente processo de desospitalização, o domicílio assume o papel de uma microunidade de saúde, na qual os profissionais que atuam na atenção primária à saúde são responsáveis por grande parte da sua organização e logística de funcionamento. Ao prover o domicílio, há que se refletir acerca do processo de retorno dos insumos ofertados, o que inclui os resíduos resultados dos cuidados na assistência à saúde. Tal 
logística é complexa e exige capacitação dos profissionais e dos usuários e/ou cuidados; estrutura física e de recursos materiais e humanos, além disso, transporte adequado que não estão previstos nas legislações atuais (ALVES SB, 2012). Assim, faz-se essencial o desenvolvimento de ações resolutivas de educação em saúde no tratamento de indivíduos com DM, de forma que elas abranjam a orientação específica acerca da doença, sendo responsabilidade da equipe multidisciplinar a atribuição de educar e intensificar a informação sobre as estratégias corretas de eliminação dos resíduos (CUNHA GHD, et al., 2017).

Nessa discussão, a educação ambiental destaca-se como a principal estratégia para o processo de conscientização e transformação de comportamentos da população, devendo assim, ser realizada de maneira periódica. Outro ponto relevante a ser considerado, é a educação permanente dos trabalhadores, uma vez que os trabalhadores que atuam nas unidades básicas de saúde precisam estar habilitados para orientar os usuários e a comunidade que faz parte do território de abrangência da unidade básica de saúde acerca das formas de manejo dos RSSs (SANTOS LN e RUIZ JB, 2020).

O enfrentamento da situação social do manejo incorreto dos resíduos nos domicílios deve buscar abranger e expandir a participação coletiva dos gestores da área da saúde e meio ambiente, profissionais de saúde, coletores urbanos e comunidade em debates e discussões que acarretem propostas de construção de políticas públicas direcionadas às singularidades do manejo dos resíduos de serviços de saúde nos domicílios (ALVES SB, 2012).

Ainda que a legislação específica atual defina as normas para o processo de gerenciamento dos RSSs, fazem-se presentes lacunas em relação à responsabilização pelo manejo adequado dos RSSs produzidos no cuidado e autocuidado que ocorrem em nível domiciliar, o que determina a adoção de melhores práticas pelos pacientes. Ademais, a ausência de conceituações objetivas acerca da temática repercute na heterogeneidade das informações e orientações presentes no meio profissional e também nas informações e oferecidas por esses profissionais aos pacientes (ESTEQUI JG, et al., 2018).

Portanto, os RSSs devem ser segregados no momento e ambiente de sua produção, em consonância com as variáveis apresentadas, as quais podem ser: químicas, físicas, biológicas e riscos associados. Devem ainda, ser armazenados em recipientes que impeçam vazamentos e forneçam resistência a punctura e rompimento, e a posteriori, encaminhados até o ambiente destinado ao acondicionamento pontual ou acondicionamento externo, com o objetivo de apresentação para o processo de coleta. Os pacientes devem ser orientados e educados a descartar objetos perfurocortantes em recipientes que igualmente apresentem resistência exigida e encaminhados de maneira segura aos serviços de saúde para o descarte adequado, bem como devem ser orientados acerca dos riscos de saúde relacionados às agulhas usadas (GOVENDER D, et al., 2012).

\section{CONSIDERAÇÕES FINAIS}

Os resultados permitem concluir que não há manejo correto de resíduos da insulinoterapia produzidos pelos indivíduos com diabetes mellitus no contexto dos serviços de atenção básica à saúde de forma geral. A ausência de orientações oportunas e adequadas para os usuários e/ou cuidadores é fator determinante para que os resíduos sejam desprezados em lixo de uso doméstico ou em outros locais inadequados, o que acarreta riscos à saúde pública, profissional e ambiental. São necessários esforços dos gestores de saúde e ambientais, comunidade e profissionais para a construção de estratégias de gerenciamento correto dos resíduos tanto em curto quanto a longo prazo.

\section{REFERÊNCIAS}

1. ALVES SB. Manejo de resíduos gerados na assistência domiciliar pela Estratégia de Saúde da Família. Rev Bras Enferm, 2012; 65(1): 128-34.

2. AMERICAN DIABETES ASSOCIATION (ADA). Diagnosis and classification of diabetes mellitus. Diabetes Care, 2013; 36(1):67-74. 
3. ANDRÉ SCS, TAKAYANAGUI AMM. Orientações sobre o descarte de resíduos gerados em domicílios de usuários de insulina. Rev Baiana de Saúde Pública, 2015; 39(1):105-18.

4. AQUINO S, et al. Percepção de diabéticos e papel dos Profissionais de Saúde Sobre a Educação Ambiental de Resíduos perfurocortantes produzidos em domicílios. Revbea. 2019; 14(1): 186-206.

5. BOAS LCG, et al. Adherence to treatment for diabetes mellitus: validation of instruments for oral antidiabetics and insulin. Rev Latino-Am Enfermagem, 2014; 22(1):11-8.

6. BRASIL. Conselho Nacional do Meio Ambiente. Resoluções do CONAMA: Resoluções vigentes publicadas entre setembro de 1984 e janeiro de 2012. Brasília: Ministério do Meio Ambiente, 2012.

7. BRASIL. Ministério da Saúde. Departamento de Atenção Básica. Secretaria de Atenção à Saúde. Estratégias para o cuidado da pessoa com doença crônica: diabetes mellitus. Cadernos de Atenção Básica. Brasília: Ministério da Saúde, 2013.

8. BRASIL. Resolução da Diretoria Colegiada oㅜ 306 de 07 de Dezembro de 2004. Dispõe sobre o regulamento técnico para o gerenciamento de resíduos de serviços de saúde. Diário Oficial da República Federativa do Brasil, Brasília (DF), 10 de dezembro de 2004.

9. CHAGAS IA, et al. Patients' knowledge of diabetes five years after the end of an educational program. Rev Esc Enfrem USP, 2013; 47(5):1137-42.

10. CUNHA GHD, et al. Insulin therapy waste produced in the households of people with diabetes monitored in Primary Care. Rev Bras Enferm, 2017; 70(3): 618-25.

11. ESTEQUI JG, et al. Resíduos gerados por usuários de insulina em domicílio. REME - Rev Min Enferm. 2018; 22(1): 1-6.

12. GOLD K. Analysis: the impact of needle, syringe and lancet disposal in the community. J Diabetes Sci Technol. 2011; $5(4): 848-50$.

13. GOVENDER D, et al. Sharps disposal practices among diabetic patients using insulin. S Afr Med J, 2012; 102(3): 1634.

14. INTERNATIONAL DIABETES FEDERATION (IDF). Diabetes. 2015. Disponível em: <http://www.diabetesatlas.orgl/>. Acessado em: 21 de março de 2021.

15. ISHTIAQ O, et al. Disposal of syringes, needles, and lancets used by diabetic patients in Pakistan. J Infectar Saúde Pública, 2012; 5 (2): 182-8.

16. OURO K. Analysis: the impact of needle, syringe, and lancet disposal on the community. J Diabetes Sci Technol. Journal of Diabetes Science and Technology, 2011; 5 (4): 848-50.

17. PAIVA PA, et al. Gerenciamento de resíduos sólidos gerados na Estratégia Saúde da Família. RETEP - Rev. Tendên. da Enferm. Profis, 2017; 9(3): 2234-37.

18. RAMOS YS, et al. Vulnerabilidade no manejo dos resíduos de serviço de saúde de João Pessoa (PB, Brasil). Ciências \& Saúde Coletiva. 2011; 6(8): 3553-60.

19. SANTOS LN, RUIZ JB. Caracterização e quantificação dos resíduos perfurocortantes gerados por diabéticos do município de Umuarama, PR, Brasil. Ciência \& Saúde Coletiva, 2020; 25(7): 2813-2819.

20. SANTOS RDT, et al. Descarte residencial de resíduos de insulinoterapia no Município de Juazeirinho, Paraíba. Revista Interdisciplinar em Saúde, Cajazeiras. 2020; 7(1): 537-50.

21. SANTOS TNL, et al. Resíduos de saúde nos domicílios de pacientes com diabetes mellitus. R. Laborativa. 2019; 8(1): 44-56.

22. SILVA ÉNSF, et al. Descarte de Seringas e agulhas por pacientes com diabetes mellitus. Rev Enferm Contemporânea, 2013; 2(1): 82-102.

23. SOCIEDADE BRASILEIRA DE DIABETES (SDB). Diretrizes Da Sociedade Brasileira De Diabetes: São Paulo: Ac Farmacêutica, 2015.

24. SOCIEDADE BRASILEIRA DE DIABETES (SDB). Diretrizes da Sociedade Brasileira de Diabetes. 2017. Disponível em: https://www.diabetes.org.br/profissionais/images/DIRETRIZES-COMPLETA-2019-2020.pdf. Acessado em: 21/3/2021.

25. STACCIARINI TSG, et al. Distribuição e utilização de seringas para aplicação de insulina na Estratégia Saúde da Família. Rev Eletr Enf, 2010; 2(1): 47-55.

26. TAPIA CEV. Diabetes Mellitus e o descarte de seringas e agulhas. Rev Gaúcha Enferm, 2009; 30(2): $228-34$.

27. TORRES HC, et al. Home visit: an educational health strategy for self-care in diabetes. Acta Paul Enferm. 2014; 27(1): 23-8.

28. URSI ES. Prevenção de lesões de pele no perioperatório: revisão integrativa da literatura. Dissertação (Mestrado em Enfermagem) - Escola de Enfermagem. Universidade de São Paulo, Ribeirão Preto, 2005; 130 p.

29. VAZ KV, et al. Investigação sobre forma de descarte de medicamentos vencidos. Cenarium Pharmacêutico 2011; $4(4): 3-27$.

30. VIEIRA M, PADILHA MICS. O HIV e o trabalhador de enfermagem frente ao acidente com material perfurocortante. Rev Esc Enferm USP 2008; 42(4): 804-810. 